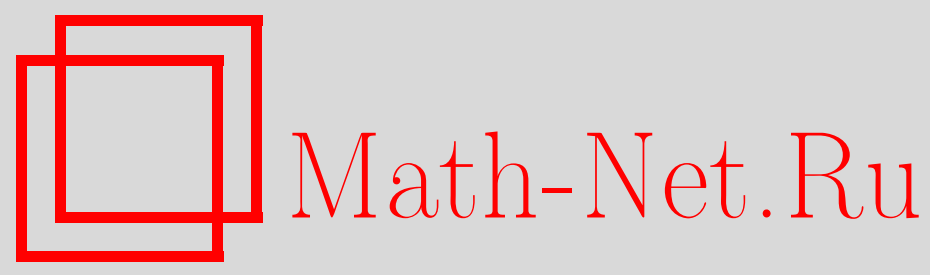

В. А. Побережный, О специальных группах монодромии и проблеме Римана-Гильберта для уравнения Римана, $M a$ тем. заметки, 2005, том 77, выпуск 5, 753-767

DOI: https://doi.org/10.4213/mzm2532

Использование Общероссийского математического портала Math-Net.Ru подразумевает, что вы прочитали и согласны с пользовательским соглашением http://www.mathnet.ru/rus/agreement

Параметры загрузки:

IP : 3.85 .73 .92

26 апреля 2023 г., 13:53:06

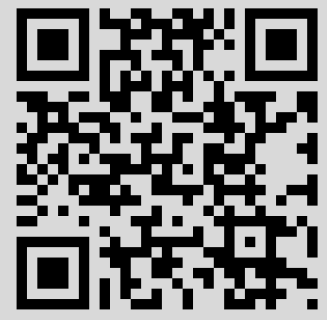




\section{О СПЕЦИАЛЬНЫХ ГРУППАХ МОНОДРОМИИ И ПРОБЛЕМЕ РИМАНА-ГИЛЬБЕРТА ДЛЯ УРАВНЕНИЯ РИМАНА}

\section{В. А. Побережный}

В работе дано решение проблемы Римана-Гильберта для уравнения Римана и гипергеометрического уравнения. Описаны все возможные представления монодромии уравнения Римана. Показано, что если монодромия уравнения Римана лежит в $S L(2, \mathbb{C})$, то ее можно реализовать не только уравнением Римана, но и более специальным уравнением Римана-Штурма-Лиувилля. Для гипергеометрического уравнения нами построен критерий принадлежности группы его монодромии к $S L(2, \mathbb{Z})$.

Библиографоия: 10 названий.

Уравнением Римана назьвают фуксово дифференциальное уравнение второго порядка с тремя особыми точками $\mathscr{D}=\left\{a_{1}, a_{2}, a_{3}\right\}$ на сфере Римана. Как известно, всякое уравнение порождает некоторое представление монодромии. А именно, взяв в точке $z_{0}$ некоторый росток фундаментального набора решений $Y(z)$ и продолжив его вдоль петли $\gamma$ получим другой росток $Y^{*}(z)$, который также является ростком некоторого базиса и связан с $Y(z)$ постоянной матрицей: $Y(z)=Y^{*}(z) G_{\gamma}$. Очевидно, $G_{\gamma}$ зависит не от самого пути $\gamma$, а лишь от гомотопического класса $[\gamma]$. Определенный таким образом гомоморфизм $\chi: \pi_{1}(\overline{\mathbb{C}} \backslash \mathscr{D}) \rightarrow G L(2, \mathbb{C})$ назьвается представлением монодромии, а его образ - әруппой монодромии уравнения, или просто монодромией. Задача восстановления уравнения по представлению монодромии была впервые поставлена Риманом. Вопрос о существовании фуксова линейного дифференциального уравнения с заданными монодромией и особыми точками был включен Гильбертом под номером 21 в число его математических проблем, и носит название проблемы Римана-Гильберта. В данной работе исследуется монодромия уравнения Римана и решается для него проблема Римана-Гильберта. Известно, что фуксово уравнение порядка $p$ с $n$ особыми точками задается

$$
\frac{p^{2}}{2}(n-2)+\frac{p n}{2}
$$

параметрами. Представление же монодромии зависит от $p^{2}(n-2)+1$ констант. Следовательно, при $p>2$ и $n>3$ построение уравнения по заданной монодромии в общем случае невозможно, так как представление задается большим числом параметров, чем

Работа выполнена при частичной поддержке Российского фонда фундаментальных исследований, грант № 01-01-00539, программы поддержки ведущих научных школ, грант № НШ-457.2003.1, и программы "Математические методы в нелинейной динамике". 
уравнение. Таким образом, случай уравнения Римана $(p=2, n=3)$ является выделенным.

В первой части работы с помощью результатов Левеля описаны представления монодромии, которые может иметь уравнение Римана. Во второй части показано, что всякую монодромию из $S L(2, \mathbb{C})$, которая может быть реализована уравнением Римана, можно реализовать и более специальным уравнением Римана-Штурма-Лиувилля. В третьей части построен критерий принадлежности монодромии гипергеометрического уравнения группе $S L(2, \mathbb{Z})$. Результат первой части был ранее получен в [1] алгебраическими методами с помощью теории представлений и соотношения $\Phi$ укса.

Автор признателен А. А. Болибруху за внимание к работе, ценные обсуждения, критические замечания и поддержку.

\section{0. Предварительные сведения}

В этом разделе мы изложим некоторые факты из теории фуксовых уравнений и систем, которые нам потребуются в дальнейшем. Так как сначала нас интересует локальная теория, для удобства будем считать нуль одной из особых точек.

ОПРЕДЕЛЕНИЕ 1. Уравнение

$$
y^{(k)}+b_{1}(z) y^{(k-1)}+\cdots+b_{k}(z) y=0
$$

назьвается фуксовым в особой точке нуль, если $b_{j}(z)$ имеет в нуле полюс порядка не более $j$, т.е. $b_{j}(z) z^{j}$ голоморфна в окрестности точки 0 .

ОПРЕДЕЛЕНИЕ 2. Система

$$
\dot{y}=B(z) y, \quad y \in \mathbb{C}^{n}, \quad B(z) \in M_{n}(\mathbb{C})
$$

назьвается фуксовой в особой точке нуль (особая точка нуль системы фуксова), если $B(z)$ имеет там полюс первого порядка.

ОПРЕДЕЛЕниЕ 3. Система (уравнение) назьвается фуксовой, если оно фуксово во всех своих особых точках.

ОПРЕДЕЛЕниЕ 4. Особая точка нуль для системы (уравнения) называется регулярной, если у любой компоненты решения рост в ней при стремлении $z$ к нулю по сектору с центром в нуле, раствора меньше $2 \pi$ не более, чем степенной.

Известна связь понятий регулярности и фуксовости для уравнений [3], [4].

УТВЕРЖДЕНИЕ 1. Уравнение регулярно тогда и только тогда, когда оно фуксово.

Для систем имеем [4], [5]

УТВЕРЖДЕНИЕ 2. Если система фуксова, то она регулярна.

Определим теперь понятие левелевского нормирования в точке $a_{i}$. 
ОПРЕДЕЛЕНИЕ 5. Левелевское нормирование в точке нуль для функции $f(z)$ определяется следующим образом:

$$
\varphi_{0}(f)=\sup \left(\lambda \in \mathbb{Z}: \frac{f(z)}{z^{\lambda}} \rightarrow 0 \text { при } z \rightarrow 0 \text { по сектору раствора меньше } 2 \pi\right) .
$$

Нормирования вектора и матрииы определяются как минимум нормирований компонент.

В частности, если $f(z)=c_{-k} / z^{k}+\cdots+c_{0}+\cdots+c_{j} z^{j}+\cdots$, то $\varphi_{0}(f)=-k$.

Для введенного нормирования выполняются следующие свойства:

1) $\varphi_{0}(c \cdot f)=\varphi_{0}(f)$ для любого $c \in \mathbb{C}$;

2) $\varphi_{0}\left(f \cdot \ln ^{\alpha} z\right)=\varphi_{0}(f)$ для любого $\alpha \in \mathbb{C}$;

3) $\varphi_{0}\left(f_{1}+f_{2}\right) \geqslant \min \left(\varphi_{0}\left(f_{1}\right), \varphi_{0}\left(f_{2}\right)\right)$ и при $\varphi_{0}\left(f_{1}\right) \neq \varphi_{0}\left(f_{2}\right)$ имеет место строгое равенство;

4) $\varphi_{0}\left(g^{*} f\right)=\varphi_{0}(f)$, где $g^{*}$ - оператор монодромии, соответствуюший обходу вокруг нуля против часовой стрелки.

Введенное нормирование определяет подпространства $\mathscr{X}^{j}$ пространства решений $\mathscr{X}$ :

$$
\mathscr{X}^{j}=\left\{f \in \mathscr{X}: \varphi_{0}(f) \geqslant j\right\},
$$

для которых при $i \geqslant k \quad \mathscr{X}^{i} \subseteq \mathscr{X}^{k}$. Из конечномерности $\mathscr{X}$ сразу же получаем, что нормирование на $\mathscr{X}$ принимает лишш конечное число значений $-\infty<\varphi_{0}^{1}<\cdots<\varphi_{0}^{k}<\infty$, и определяет левелевскую фильтрацию:

$$
0 \subset \mathscr{X}^{\varphi_{k}} \subset \cdots \subset \mathscr{X}^{\varphi_{1}}=\mathscr{X} .
$$

Из свойств нормирования следует, что все $\mathscr{X}^{\varphi_{j}}$ инвариантны относительно действия оператора соответствующей монодромии.

ОПРЕДЕЛЕНИЕ 6 . Базис $\left(e_{1}, \ldots, e_{p}\right)$ пространства решений системы или уравнения назьвается слабо ассочиированным в точке $a_{i}$, если на нем $\varphi_{a_{i}}(\cdot)$ принимает все свои возможные значения с учетом кратностей.

ОПРЕДЕЛЕНИЕ 7. Слабо ассоциированньй базис $\left(e_{1}, \ldots, e_{p}\right)$ назьвается ассоциированным, если матрища монодромии $G_{a_{i}}$ в нем верхнетреугольна и, кроме того, $\varphi_{a_{i}}\left(e_{k}\right) \geqslant$ $\varphi_{a_{i}}\left(e_{k+1}\right)$.

ОПРЕДЕЛЕниЕ 8. Базис, полученный объединением ассоциированных базисов корневых подпространств оператора монодромии, называется левелевским.

УТВЕРЖДЕНИЕ 3 [6]. Левелевский базис является слабо ассоциированным.

ОПредЕлЕниЕ 9. Пусть $G$-матрица монодромии. Тогда ее нормализованный логарифм есть матрища $E$ следующего вида:

$$
E=\frac{1}{2 \pi i} \ln G, \quad \operatorname{Re} \rho_{i} \in[0 ; 1),
$$

где $\rho_{i}-$ собственные значения $E, \operatorname{a} \operatorname{Re} \rho_{i}$ их целые части.

Легко заметить, что при обходе вокруг нуля $z^{-E_{0}} \rightarrow G_{0}^{-1} z^{-E_{0}}$ и, таким образом, фундаментальная матрица в окрестности нуля допускает разложение $Y=M(z) z^{E_{0}}$. Здесь $M(z)$ - мероморфная матричная функция, а $E_{0}$ - соответствующий нормализованньй логарифм. 
УТВЕРЖДЕНИЕ 4 [7]. В слабо ассочиированном базисе фундаментальная матрича системы с регулярной особой точкой 0 допускает разложсние

$$
Y(z)=U_{0}(z) z^{A_{0}} z^{E_{0}}
$$

где $E_{0}$ - нормализованный логарифм матрицы $G_{0}, A_{0}$ - иелочисленная диагональная матрица нормирований столбиов $A_{0}=\operatorname{diag}\left(\varphi_{0}^{1}, \ldots \varphi_{0}^{p}\right), U_{0}(z)$ голоморфна в окрестности нуля. Система фуксова в нуле тогда и только тогда, когда $\operatorname{det} U_{0}(0) \neq 0$.

ОПРЕДЕЛЕНИЕ 10. Числа $\beta_{i}^{j}=\varphi_{i}^{j}+\rho_{i}^{j}$, стоящие на диагонали матрицы $A_{a_{i}}+E_{a_{i}}$, полученной разложением матрицы ассоциированного в $a_{i}$ базиса, называются показателями пространства $\mathscr{X}$ в точке $a_{i}$.

УТВЕРЖДЕНИЕ 5 [6]. Сумма показателей системы с регулярными особыми точками - неположительное челое число; система фуксова тогда и только тогда, когда $\sum_{\mathscr{D}} \sum_{j=1}^{j=p} \beta_{i}^{j}=0$.

УТВЕРЖДЕНИЕ 6 [8]. Для показателей фуксова уравнения порядка р с $п$ особыми точками имеет место соотношение Фукса

$$
\sum_{\mathscr{D}} \sum_{j=1}^{j=p} \beta_{i}^{j}=\frac{(n-2) p(p-1)}{2}
$$

Задача восстановления системы по заданной монодромии носит название проблемы Римана-Гильберта. Данная работа основывается на следующем результате, полученном Крыловым [9].

УТВЕРЖДЕНИЕ 7. В размерности 2 проблема Римана-Гильберта разрешима в классе фуксовых систем с тремя особьми точками. То есть всегда существует фуксова система с тремя заранее заданными особыми точками, реализующая требуемую монодромию.

Аналогичный результат для произвольного числа точек был позднее получен Деккерсом [10].

\section{1. $G L$-монодромия}

1.1. Неприводимые представления. Пусть задано неприводимое $G L(2, \mathbb{C})$-представление $\chi$ и $Y$ - фундаментальная матрища решений системы, реализующая $\chi$ (по утверждению 7 такая система существует), построенная по левелевскому базису в точке $a_{i}$

$$
Y(z)=U_{i}(z) z^{A_{i}} z^{E_{i}}, \quad \operatorname{det} U_{i}\left(a_{i}\right) \neq 0
$$

Рассмотрим теперь

$$
\tilde{Y}=\left(\begin{array}{ll}
0 & 1 \\
1 & 0
\end{array}\right) U_{i}^{-1}\left(a_{i}\right) Y .
$$

Очевидно, $\tilde{Y}$ - фундаментальная матрица системы с теми же особьми точками и монодромией. Обозначим ее опять за $Y$. 
Лемма 1.1. В случае неприводимости представления $\chi$ әлементы первой строки матрицы $Y(z)$ - функиии $y_{1}(z), y_{2}(z)$ - линейно независимы.

ДокАЗАТЕЛЬСтво. Предположим, что $y_{1}(z), y_{2}(z)$ зависимы, т.е.

$$
y_{2}(z)=k y_{1}(z)
$$

Это накладывает следующие условия на матрицы $G_{a_{i}}$ : так как из $(1)$ следует $g_{i}^{*}\left(y_{2}\right)=$ $k g_{i}^{*}\left(y_{1}\right)$ для всех $i$, где $g_{i}^{*}$ - оператор монодромии, соответствующий обходу $a_{i}$, то для $G_{a_{i}}$, имеюших вид

должно вьполняться

$$
G_{a_{i}}=\left(\begin{array}{cc}
\alpha_{i} & \beta_{i} \\
\gamma_{i} & \delta_{i}
\end{array}\right)
$$

$$
k \alpha_{i}+k^{2} \gamma_{i}=\beta_{i}+k \delta_{i}
$$

Известно,что фундаментальную матрицу решений можно умножать справа на невырожденную, получая опять фундаментальную матрицу. Это соответствует замене базиса в пространстве решений. Взяв теперь

$$
S=\left(\begin{array}{cc}
k & 0 \\
1 & 1
\end{array}\right), \quad S^{-1}=\left(\begin{array}{cc}
k^{-1} & 0 \\
-k^{-1} & 1
\end{array}\right),
$$

получим в базисе $\widetilde{Y}=Y S$

$$
\widetilde{G}_{a_{i}}=S^{-1} G_{a_{i}} S=\left(\begin{array}{cc}
\alpha_{i}-k^{-1} \beta_{i} & k^{-1} \\
0 & \delta_{i}-k^{-1} \beta_{i}
\end{array}\right)
$$

для всех $i$ одновременно, что противоречит неприводимости представления $\chi$.

Итак, $Y$ - матрица, построенная по левелевскому базису, такая, что

$$
Y=\left(\begin{array}{ll}
y_{1} & y_{2} \\
y_{3} & y_{4}
\end{array}\right), \quad U_{i}\left(a_{i}\right)=\left(\begin{array}{ll}
0 & 1 \\
1 & 0
\end{array}\right) .
$$

Возьмем теперь $\left(y_{1}, y_{2}\right)$ за базис пространства решений некоторого фуксова уравнения и покажем, что оно будет уравнением Римана. Из вида $U_{i}\left(a_{i}\right)$ следует $\varphi_{a_{i}}\left(y_{1}\right) \geqslant \varphi_{a_{i}}^{1}$. Показатели уравнения в точках $\mathscr{D}$ очевидным образом связаны с показателями исходной системы:

$$
\begin{gathered}
\tilde{\beta}_{i}^{1} \geqslant \beta_{i}^{1}+1, \quad \tilde{\beta}_{i}^{2}=\beta_{i}^{2}, \\
\operatorname{Re} \tilde{\beta}_{j}^{k} \geqslant \operatorname{Re} \beta_{j}^{k}, \quad \operatorname{Im} \tilde{\beta}_{j}^{k}=\operatorname{Im} \beta_{j}^{k}, \quad j \neq i .
\end{gathered}
$$

ЛЕмма 1.2. Пусть $\left(\tilde{y}_{1}, \tilde{y}_{2}\right)$ - независимые, голоморфные на $(\overline{\mathbb{C}} \backslash \mathscr{D})$ әлементы первой строки некоторой фундаментальной матрицы системы с неприводимой монодромией, D содержит не более трех точек и выполняется $\sum_{\mathscr{D}}\left(\tilde{\beta}_{i}^{1}+\tilde{\beta}_{i}^{2}\right) \geqslant 1$. Тогда

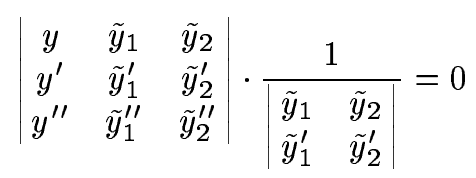

- фуксово уравнение с особыми точками $\mathscr{D}$. 
ДокАЗАТЕльство. Уравнение будет регулярно и, следовательно, фуксово. Предположим, что благодаря нулям детерминанта у уравнения появится $k$ ложных особых точек $\mathscr{D}_{k}$. Тогда из соотношения Фукса (утверждение 6)

$$
\sum_{\mathscr{D}}\left(\tilde{\beta}_{i}^{1}+\tilde{\beta}_{i}^{2}\right)+\sum_{\mathscr{D}_{k}}\left(\tilde{\beta}_{i}^{1}+\tilde{\beta}_{i}^{2}\right) \leqslant k+1 .
$$

Заметим, что в точках $\mathscr{D}_{k}$ решения голоморфны и, следовательно, показатели в них целые неотрицательные числа. Кроме того, определитель, стоящий в знаменателе, равен нулю в некоторой точке тогда и только тогда, когда существует линейная комбинация $\tilde{y}_{1}$, $\tilde{y}_{2}$, имеющая в данной точке нуль, по крайней мере, второго порядка (т.е. когда столбцы линейно зависимы). Следовательно, в любой точке $b_{i}$, принадлежащей $\mathscr{D}_{k}$, хотя бы одно из нормирований, а следовательно, и показателей больше или равно 2 . Тогда

$$
1+k \geqslant \sum_{\mathscr{D}}\left(\tilde{\beta}_{i}^{1}+\tilde{\beta}_{i}^{2}\right)+\sum_{\mathscr{D}_{k}}\left(\tilde{\beta}_{i}^{1}+\tilde{\beta}_{i}^{2}\right) \geqslant 1+2 k,
$$

что возможно только при $k=0$. То есть уравнение не имеет дополнительных особых точек.

СлЕДСТВИЕ 1.1. Уравнение, построенное по выбранным выше $y_{1}, y_{2}$, будет искомым уравнением Римана.

ДокАЗАТЕЛЬСТво. Из выбора $y_{1}, y_{2}$ следует, что уравнение будет фуксовым; по доказанной лемме оно будет иметь ровно три особых точки; следовательно, - это уравнение Римана.

СлЕДСТВИЕ 1.2. Показатели построенного таким образом уравнения связань с показателями исходной системы следующим образом:

$$
\begin{gathered}
\tilde{\beta}_{j}^{k}=\beta_{j}^{k} \quad \text { npu } j \neq i, \\
\tilde{\beta}_{i}^{1}=\beta_{i}^{1}+1, \quad \tilde{\beta}_{i}^{2}=\beta_{i}^{2} .
\end{gathered}
$$

Таким образом, мы доказали центральную теорему этого раздела.

ТЕоремА 1.1. Любое неприводимое двумерное представление монодромии $с$ двумя независимыми порожсдающими реализуется фуксовым уравнением второго порядка с тремя особыми точками.

1.2. Приводимые представления. Так как мы рассматриваем приводимые представления, задающиеся всего двумя матрищами, нам надо рассмотреть всего три случая. А именно, в зависимости от того ни одна из матриц, одна или две имеют диагональный жорданов вид, будем называть соответствующие представления недиагонализуемымми, диагонализуемыми или диагональными.

1.2.1. Диагональная монодромия. Имеется ввиду случай, приводящийся к $G_{i}=$ $\operatorname{diag}\left(\alpha_{i}, \beta_{i}\right)$ для всех $i$ одновременно. Прежде всего докажем следующую лемму.

Лемма 1.3. Если уравнение Римана имеет диагональную монодромию, то хотя бъ одна из $G_{i}-$ скалярная матрица. 
ДокАЗАТЕЛьСтво. Предположим существует уравнение с диагональной монодромией без скалярных матриц. Проделаем с ним следующие преобразования. Положим $a_{3}=\infty$, что всегда можно достичь конформным преобразованием сферы Римана. Если все матрищы не скалярны, то существуют два независимых решения, образующих глобальньй левелевский базис:

$$
u_{1}(z)=\left(z-a_{1}\right)^{\beta_{1}^{1}}\left(z-a_{2}\right)^{\beta_{2}^{1}} P_{1}(z), \quad u_{2}(z)=\left(z-a_{1}\right)^{\beta_{1}^{2}}\left(z-a_{2}\right)^{\beta_{2}^{2}} P_{2}(z),
$$

где $\beta_{i}^{1} \neq \beta_{i}^{2}, P_{1}, P_{2}$ - многочлены степеней $n_{1}, n_{2}$ без нулей в $a_{1}, a_{2}$. Так как оператор монодромии в $\infty$ не скалярен, то $\beta_{3}^{j}=-\beta_{1}^{j}-\beta_{2}^{j}-n_{j}\left(\left(u_{1}, u_{2}\right)\right.$ - глобальньй левелевский базис). Напомним, что если все собственные значения монодромии различны, как в нашем случае, то левелевский базис определен однозначно. Но по соотношению Фукса

$$
\sum_{\mathscr{D}}\left(\beta_{i}^{1}+\beta_{i}^{2}\right)=-n_{1}-n_{2}=1
$$

что невозможно.

Итак можно считать, что $G_{3}=\gamma \cdot I$. Выберем $y_{1}, y_{2}-$ решения фуксовых уравнений первого порядка с монодромиями $\alpha_{1}, \beta_{1}, \gamma$ и $\alpha_{2}, \beta_{2}, \gamma$ :

$$
y_{1}=\left(z-a_{1}\right)^{\lambda_{1}}\left(z-a_{2}\right)^{\mu_{1}}\left(z-a_{3}\right)^{\nu}, \quad y_{2}=\left(z-a_{1}\right)^{\lambda_{2}}\left(z-a_{2}\right)^{\mu_{2}}\left(z-a_{3}\right)^{\nu}
$$

так, чтобы они удовлетворяли соотношению $\lambda_{i}+\mu_{i}+\nu=0$ (теперь мы уже полагаем $\infty$ точкой голоморфности), что всегда возможно, так как их сумма целая $\left(e^{2 \pi i\left(\lambda_{i}+\mu_{i}+\gamma\right)}=\right.$ $\left.\alpha_{i} \beta_{i} \gamma=1\right)$, а монодромией каждое из чисел также определяется лишь с точностью до целого. По этой же причине $y_{1}, y_{2}$ можно считать линейно независимьми. Возьмем теперь $y_{1}, y_{2}$ за базис пространства решений некоторого уравнения. Очевидно, для этого уравнения показатели в точках $a_{1}, a_{2}, a_{3}$ соответственно равны $\left(\lambda_{1}, \lambda_{2}\right),\left(\mu_{1}, \mu_{2}\right)$, $(\nu+1, \nu)$. Действительно, так как в $a_{3}$ и $y_{1}$, и $y_{2}$ пропорциональны $\left(z-a_{3}\right)^{\nu}$, существует их линейная комбинация, растущая как $\left(z-a_{3}\right)^{\nu+1}$. Теперь из леммы 1.2 сразу же получаем, что данное уравнение будет уравнением Римана. Таким образом, доказана

Теорема 1.2. Диагональное представление реализуется уравнением Римана тогда и только тогда, когда хотя бы одна из матрии монодромии скалярна.

1.2.2. Диагонализуемая в точке монодромия. Пусть задано приводимое неразложимое представление

$$
\chi: \quad G_{1}=\left(\begin{array}{cc}
g_{11}^{1} & 0 \\
0 & g_{22}^{1}
\end{array}\right), \quad G_{2}=\left(\begin{array}{cc}
g_{11}^{2} & g_{12}^{2} \\
0 & g_{22}^{2}
\end{array}\right), \quad G_{3}=\left(\begin{array}{cc}
g_{11}^{3} & g_{12}^{3} \\
0 & g_{22}^{3}
\end{array}\right)
$$

Лемма 1.4. Пусть $Y$ - фундаментальная матрица системы с заданной монодромией. Тогда пары $\left(y_{11}, y_{12}\right)$ и $\left(y_{21}, y_{22}\right)$ не могут быть линейно зависимыми одновременно. 
ДокАЗАТЕЛЬСТво. Предположим $k \cdot y_{11}=y_{12}, y_{21}=l \cdot y_{22}$. Здесь $k l \neq 1$, так как столбцы линейно независимы. Тогда в базисе

$$
\tilde{Y}=Y\left(\begin{array}{cc}
1 /(1-k l) & -k /(1-k l) \\
-l /(1-k l) & 1 /(1-k l)
\end{array}\right)=\left(\begin{array}{cc}
\tilde{y}_{1} & 0 \\
0 & \tilde{y}_{4}
\end{array}\right)
$$

фундаментальная матрица и, следовательно, монодромия диагональны, что противоречит неразложимости представления $\chi$.

Итак, пусть $Y$ - левелевская в точке с диагональной монодромией фундаментальная матрица. Пусть это точка $a_{1}$. Перейдем к

$$
\tilde{Y}=\left(\begin{array}{ll}
0 & 1 \\
1 & 0
\end{array}\right) U_{1}^{-1}\left(a_{1}\right) Y
$$

и обозначим ее снова за $Y$. Для этой матрищы

$$
U_{1}\left(a_{1}\right)=\left(\begin{array}{ll}
0 & 1 \\
1 & 0
\end{array}\right)
$$

теперь либо $\left(y_{11}, y_{12}\right)$, либо $\left(y_{21}, y_{22}\right)$ линейно независимы, и являются либо левелевским базисом уравнения с натянутым на них пространством решений, либо его транспозицией (так как $G_{0}$ диагональна) и, следовательно, удовлетворяя условиям леммы 1.2 , порождают искомое уравнение Римана. Отсюда вытекает

Теорема 1.3. Всякое приводимое неразложимое представление, диагонализуемое хотя бы в одной точке, мохет быть реализовано уравнением Римана.

1.2.3. Недиагонализуемая монодромия. Речь пойдет о представлениях вида

$$
\chi: \quad G_{1}=\left(\begin{array}{cc}
\lambda_{1} & 1 \\
0 & \lambda_{1}
\end{array}\right), \quad G_{2}=\left(\begin{array}{cc}
\lambda_{2} & c \\
0 & \lambda_{2}
\end{array}\right), \quad G_{3}=\left(\begin{array}{cc}
\lambda_{3} & d \\
0 & \lambda_{3}
\end{array}\right)
$$

Базис $\left(y_{1}, y_{2}\right)$, в котором монодромия имеет такой вид, будет глобальным левелевским базисом, так как единственное подпространство, инвариантное относительно оператора, приводящегося к жордановой клетке, натянуто на первьй вектор жорданова базиса. Следовательно, показатели уравнения можно определять по этому базису. Можно заметить, что $\beta_{i}^{1}$ есть ничто иное, как логарифмический вьчет $y_{1}$ в точке $a_{i}$. Сумма всех вычетов $y_{1}$ по $\mathbb{C} P^{1}$ равна нулю и складывается из суммы вычетов по $\mathscr{D}$ и неотрицательной суммы вычетов по нулям $y_{1}$. Таким образом, $\sum_{\mathscr{D}} \beta_{i}^{1} \leqslant 0$. Из левелевости базиса следует также $\operatorname{Re} \beta_{i}^{1} \geqslant \operatorname{Re} \beta_{i}^{2}$. Отсюда получаем $\sum_{\mathscr{D}}\left(\beta_{i}^{1}+\beta_{i}^{2}\right) \leqslant 2 \sum_{\mathscr{D}} \beta_{i}^{1} \leqslant 0$, что противоречит соотношению Фукса. Таким образом из сказанного следует

ТЕОремА 1.4. Всякое приводимое представление монодромии уравнения Римана диагонализуемо хотя бъ в одной точке. 


\section{2. $S L$-монодромия}

Известно [3], что уравнение Римана полностью определяется своим дивизором $\mathscr{D}$ и показателями $\beta_{i}^{j}$. А именно, если $\infty \notin \mathscr{D}=\left\{a_{1}, a_{2}, a_{3}\right\}$, то уравнение имеет вид

$$
y^{\prime \prime}+\left(\sum_{\mathscr{D}} \frac{1-\beta_{i}^{1}-\beta_{i}^{2}}{z-a_{i}}\right) y^{\prime}+\left(\sum_{\mathscr{D}} \frac{\beta_{i}^{1} \beta_{i}^{2}}{z-a_{i}} \cdot \frac{\prod_{j \neq i}\left(a_{i}-a_{j}\right)}{\prod_{\mathscr{D}}\left(z-a_{i}\right)}\right) y=0 .
$$

Если же $\infty \in \mathscr{D}$, то полагая $a_{3}=\infty$, получаем

$$
\begin{aligned}
y^{\prime \prime} & +\left(\sum_{i=1,2} \frac{1-\beta_{i}^{1}-\beta_{i}^{2}}{z-a_{i}}\right) y^{\prime} \\
& +\left(\frac{\beta_{1}^{1} \beta_{1}^{2}\left(a_{1}-a_{2}\right)}{z-a_{1}}+\frac{\beta_{2}^{1} \beta_{2}^{2}\left(a_{2}-a_{1}\right)}{z-a_{2}}+\beta_{3}^{1} \beta_{3}^{2}\right) \frac{y}{\left(z-a_{1}\right)\left(z-a_{2}\right)}=0 .
\end{aligned}
$$

ОПРеДЕЛЕнИЕ 2.1. Будем называть уравнением Римана-Штурма-Лиувилля уравнение Римана с нулевым коэффициентом при $y^{\prime}$ :

$$
y^{\prime \prime}+q(z) y=0
$$

Из $(2),(3)$ и соотношения Фукса на $\beta_{i}^{j}$ сразу же следует, что $\infty$ является одной из особых точек уравнения Римана-Штурма-Лиувилля. Исследуем теперь монодромию этого уравнения. Из выражения коэффициентов через показатели следует для $i=1,2$

$$
1=\beta_{i}^{1}+\beta_{i}^{2}=\left(\rho_{i}^{1}+\rho_{i}^{2}\right)+\left(\varphi_{i}^{1}+\varphi_{i}^{2}\right) \Longrightarrow\left(\rho_{i}^{1}+\rho_{i}^{2}\right) \in \mathbb{Z} \Longrightarrow 1=e^{2 \pi i\left(\rho_{i}^{1}+\rho_{i}^{2}\right)}=\operatorname{det} G_{i} .
$$

Таким образом, монодромия уравнения Римана-Штурма-Лиувилля лежит в $S L(2, \mathbb{C})$. Возникает вопрос, какие из $S L$-представлений могут быть реализованыподобным уравнением.

УТВЕРЖДЕНИЕ 2.1. Представление $\chi: \pi_{1}(\overline{\mathbb{C}} \backslash \mathscr{D}) \rightarrow S L(2, C)$ может быть реализовано монодромией уравнения Римана-Штурма-Лиувилля, если и только если существует реализующее $\chi$ уравнение Римана такое, что сумма показателей в двух его особьх точках из трех равна единице.

ДокАЗАТЕЛЬСтво. Пусть в точках $a_{1}, a_{2}$ сумма показателей равна единице. Конформно переведем $a_{3}$ в $\infty$. По формуле (3) полученное уравнение будет уравнением Римана-Штурма-Лиувилля. Обратное утверждение очевидно.

Остается выяснить, когда требуемую монодромию можно задать именно таким уравнением. Теперь, как и в части 1 , рассмотрим различные классы уравнений.

2.1. Диагональная монодромия. Реализуем сначала вырожденньй случай $G_{i}=I$ для всех $i$. Для этого возьмем

$$
y_{1}=\left(z-a_{1}\right)^{2}\left(z-a_{2}\right)^{-1}\left(z-a_{3}\right)^{-1}, \quad y_{2}=\left(z-a_{1}\right)^{-1}\left(z-a_{2}\right)^{2}\left(z-a_{3}\right)^{-1} .
$$

Как показано в разделе 1.2 .1 , это базис пространства решений некоторого уравнения Римана. По следствию $1.2 \beta_{i}^{1}+\beta_{i}^{2}=\varphi_{i}^{1}+\varphi_{i}^{2}=1$ для $i=1,2$. Из утверждения 2.1 
следует реализуемость данного представления. Далее, если не все $G_{i}$ единичные, то $\rho_{1}^{j}+\rho_{2}^{j}+\rho_{3}^{j}=1, j=1,2$, где $\rho_{i}^{j}-$ соответствующие нормализованные логарифмы. В этом случае, полагая по лемме $1.3 G_{3}= \pm I$, за $y_{1}, y_{2}$ берем

$$
\begin{aligned}
& y_{1}=\left(z-a_{1}\right)^{k+\rho_{1}^{1}} \cdot\left(z-a_{2}\right)^{-k+\rho_{2}^{1}} \cdot\left(z-a_{3}\right)^{-1+\rho_{3}}, \\
& y_{2}=\left(z-a_{1}\right)^{-k+\rho_{1}^{2}} \cdot\left(z-a_{2}\right)^{k+\rho_{2}^{2}} \cdot\left(z-a_{3}\right)^{-1+\rho_{3}} .
\end{aligned}
$$

Так как хотя бы в двух точках $G_{i} \neq I$, то в них $\beta_{i}^{1}+\beta_{i}^{2}=\rho_{i}^{1}+\rho_{i}^{2}=1$ и, следовательно, построенное по $y_{1}, y_{2}$ уравнение Римана удовлетворяет условиям утверждения 1.2. То есть любая диагональная монодромия, имеющая одну из $G_{i}$ скалярной, может быть реализована уравнением Римана-Штурма-Лиувилля.

2.2. Прочие представления. В данном разделе нам потребуются некоторые факты из теории фуксовых систем. Итак, матрица фуксовой системы, имеющая $\infty$ точкой голоморфности, очевидно, имеет вид

$$
B(z)=\sum_{\mathscr{D}} \frac{B_{i}}{z-a_{i}}, \quad \sum B_{i}=0
$$

ЛЕмма 2.1. Пусть $Y$ - фундаментальная матрица фуксовой системы $\dot{Y}=$ $B(z) Y$, построенная по левелевскому в $a_{i}$ базису

$$
Y=U_{i}(z)\left(z-a_{i}\right)^{A_{i}}\left(z-a_{i}\right)^{E_{i}}
$$

u nусть $L_{i}=\lim _{z \rightarrow a_{i}}\left(z-a_{i}\right)^{A_{i}} E_{i}\left(z-a_{i}\right)^{-A_{i}}+A_{i}$. Тогдa

$$
B_{i}=U_{i}\left(a_{i}\right) L_{i} U_{i}^{-1}\left(a_{i}\right)
$$

ДоКАЗАТЕЛЬСТВо. Очевидно, в окрестности $a_{i}$

$$
B(z)=\frac{d Y}{d z} Y^{-1}=\left(\frac{d U_{i}(z)}{d z} U_{i}^{-1}(z)+\frac{U_{i}(z)\left(A_{i}+\left(z-a_{i}\right)^{A_{i}} E_{i}\left(z-a_{i}\right)^{-A_{i}}\right) U_{i}^{-1}(z)}{z-a_{i}}\right)
$$

из чего сразу же вытекает утверждение леммы.

ЗАмечАниЕ. Если $A_{i}$ скалярна, то $L_{i}=A_{i}+E_{i}$.

Центральньм утверждением раздела, позволяющим получать системы наиболее удобного для дальнейших построений вида, является

Лемма 2.2 [2]. Для любого $\chi: \pi_{1}(\overline{\mathbb{C}} \backslash \mathscr{D} \rightarrow G L(2, \mathbb{C}))$ существует реализующая его фуксова система, нормирования которой хотя бы в двух точках имеют вид $\varphi_{i}^{1}=\varphi_{i}^{2}=0$. 
ДокАЗАТЕЛЬСтво. Предположим сначала, что существуют две точки, в которых нормирования совпадают. Например, точки $a_{1}, a_{2}$, т.е. $\varphi_{1}^{1}=\varphi_{1}^{2}=k, \varphi_{2}^{1}=\varphi_{2}^{2}=l$. В этом случае перейдем от пространства решений $\mathscr{X}$ к пространству

$$
\mathscr{X}^{\prime}=\left(\frac{\left(z-a_{3}\right)^{k+l}}{\left(z-a_{1}\right)^{k}\left(z-a_{2}\right)^{l}}\right)^{I} \mathscr{X}=\left(\begin{array}{cc}
\frac{\left(z-a_{3}\right)^{k+l}}{\left(z-a_{1}\right)^{k}\left(z-a_{2}\right)^{l}} & 0 \\
0 & \frac{\left(z-a_{3}\right)^{k+l}}{\left(z-a_{1}\right)^{k}\left(z-a_{2}\right)^{l}}
\end{array}\right) \mathscr{X}
$$

которое, очевидно, будет пространством решений системы с той же монодромией и искомыми нормированиями. Если же хотя бы в двух точках (предположим, опять в $a_{1}, a_{2}$ ) выполняется $\varphi_{i}^{1}-\varphi_{i}^{2}>0$, проделаем следующую процедуру. Перейдем сначала к

$$
\mathscr{X}^{\prime}=U_{1}^{-1}\left(a_{1}\right) \mathscr{X},
$$

а затем к

$$
\mathscr{X}^{\prime \prime}=\Gamma_{1}(z) \mathscr{X}=\left(\begin{array}{cc}
\left(z-a_{2}\right) /\left(z-a_{1}\right) & 0 \\
0 & 1
\end{array}\right) \mathscr{X}^{\prime} .
$$

Из вида $U_{1}^{\prime \prime}(z)$, очевидно, $\varphi_{1}^{1^{\prime \prime}}=\varphi_{1}^{1}-1, \varphi_{1}^{2^{\prime \prime}}=\varphi_{1}^{2}$. Рассмотрим теперь

$$
U_{2}^{\prime \prime}(z)=\left(\begin{array}{cc}
\left(z-a_{2}\right)\left(a_{0}+\cdots\right) & \left(z-a_{2}\right)\left(b_{0}+\cdots\right) \\
c_{0}+\cdots & d_{0}+\cdots
\end{array}\right) .
$$

Предположим теперь $a_{0} \neq 0$; тогда, беря

$$
\Gamma_{2}(z)=\left(\begin{array}{cc}
1 & 0 \\
-c_{0} a_{0}^{-1} /\left(z-a_{2}\right) & 1
\end{array}\right)
$$

получаем

$$
U_{2}^{\prime \prime \prime}(z)=\Gamma_{2}(z) U_{2}^{\prime \prime}(z)=\left(\begin{array}{cc}
\left(z-a_{2}\right)\left(a_{0}+\cdots\right) & \left(z-a_{2}\right)\left(b_{0}+\cdots\right) \\
\left(z-a_{2}\right)\left(c_{1}-c_{0} a_{1} a_{0}^{-1}+\cdots\right) & \left(a_{0}^{-1} \operatorname{det} U_{2}^{\prime}\left(a_{2}\right)+\cdots\right)
\end{array}\right) .
$$

Очевидно, $\varphi_{1}^{1^{\prime \prime \prime}}=\varphi_{1}^{1}-1, \varphi_{2}^{1^{\prime \prime \prime}}=\varphi_{2}^{1}+1$. Из инвариантности суммы показателей после всех проделанных преобразований получаем, что итоговая система также будет фуксовой. А из мероморфности $\Gamma_{i}(z)$ и их голоморфной обратимости на $\overline{\mathbb{C}} \backslash \mathscr{D}$ следует, что монодромия также не изменится.

Если же $a_{0}=0$, то по утверждению $4 b_{0} \neq 0$. Взяв

$$
\Gamma_{3}(z)=\left(\begin{array}{cc}
1 & 0 \\
-d_{0} b_{0}^{-1} /\left(z-a_{2}\right) & 1
\end{array}\right)
$$

таким же образом получаем $\varphi_{1}^{1^{\prime \prime \prime}}=\varphi_{1}^{1}-1, \varphi_{2}^{1^{\prime \prime \prime}}=\varphi_{2}^{2}+1$. Остальные нормирования ни в том, ни в другом случае, очевидно, не меняются. Таким образом, после проделанной процедуры хотя бы в одной точке разность между $\varphi_{i}^{1}$ и $\varphi_{i}^{2}$ уменьшилась на единицу. Повторяя подобные действия до тех пор, пока одна из разностей не станет равной нулю, и при необходимости проведя ту же процедуру для двух других точек, получаем уже рассмотренный случай, когда $\varphi_{i}^{1}=\varphi_{i}^{2}$ хотя бы в двух точках. Лемма доказана. 
ЗАмЕчАнИЕ. Использованные в доказательстве калибровочные преобразования $\Gamma_{2}(z)$ и $\Gamma_{3}(z)$ являются частными случаями более общих, построенных в работе [6] $A$ - и $B$-преобразований.

СлЕДСТвИЕ. Если $\chi$ - некоторое недиагональное SL-представление, то существует реализующая его фуксова система, для которой в двух точках $\mathscr{D}$ выполняется $\Sigma_{i}=\beta_{i}^{1}+\beta_{i}^{2}=1$.

ДокАЗАТЕЛЬСТво. Возьмем систему, реализующую $\chi$, построенную в лемме 2.2. В зависимости от $\varepsilon_{i}=\rho_{i}^{1}+\rho_{i}^{2}$ возможны следующие случаи (с точностью до перестановки).

1) $\Sigma_{1}=\Sigma_{2}=1, \Sigma_{3}=-2$ - система, удовлетворяющая условиям следствия.

2) $\Sigma_{1}=1, \Sigma_{2}=0, \Sigma_{3}=-1$. Перейдя к $\mathscr{X}^{\prime}=\Gamma(z) \mathscr{X}$, где $\Gamma(z)=\left(\left(z-a_{3}\right) /\left(z-a_{2}\right)\right)^{I}$, получим $\Sigma_{1}^{\prime}=\Sigma_{3}^{\prime}=1$.

3) $\Sigma_{1}=\Sigma_{2}=\Sigma_{3}=0$ (т.е. $\varepsilon_{1}=\varepsilon_{2}=\varepsilon_{3}=0$ ). Проведем следующее преобразование:

$$
\mathscr{X}^{\prime}=\left(\begin{array}{ll}
0 & 1 \\
1 & 0
\end{array}\right) U_{1}^{-1}\left(a_{i}\right) \mathscr{X} \text {. }
$$

Предположим, что хотя бы в одной из матриц $U_{2}^{\prime}\left(a_{2}\right), U_{3}^{\prime}\left(a_{3}\right)$ верхний левьй элемент $u_{11}^{i}\left(a_{i}\right)$ не равен нулю; тогда проводя описанную в лемме 2.2 процедуру, можно повысить, например, $\varphi_{2}^{1}$, понижая $\varphi_{2}^{1}$, после чего $\Sigma_{1}^{\prime}=-1, \Sigma_{2}^{\prime}=1, \Sigma_{3}^{\prime}=0$, и, таким образом, этот случай сведется к предыдущему. Предположив же, что $u_{11}^{i}\left(a_{i}\right)=0$ для любого $i$, получаем

$$
\begin{gathered}
U_{i}\left(a_{i}\right)=\left(\begin{array}{ll}
0 & b \\
c & d
\end{array}\right), \\
B_{i}=U_{i}\left(a_{i}\right) L_{i} U_{i}^{-1}\left(a_{i}\right)=\left(\begin{array}{ll}
0 & b \\
c & d
\end{array}\right)\left(\begin{array}{ll}
0 & \lambda \\
0 & 0
\end{array}\right)\left(\begin{array}{cc}
-d /(b c) & 1 / c \\
1 / b & 0
\end{array}\right)=\left(\begin{array}{cc}
0 & 0 \\
\lambda c / b & 0
\end{array}\right),
\end{gathered}
$$

т.е. $B(z)=\sum B_{i} /\left(z-a_{i}\right)$ имеет вид

$$
B(z)=\left(\begin{array}{cc}
0 & 0 \\
f(z) & 0
\end{array}\right) .
$$

Такая система приводима, так как существует решение

$$
y=\left(\begin{array}{l}
0 \\
c
\end{array}\right)
$$

а нуль, продолженный вдоль любого пути, дает нуль и, следовательно, $g_{21}^{i}=0$. Из доказанного в первой части следует, что в этом случае одну из $G_{i}$ можно считать диагональной. Тогда можно понизить нормирование второго столбца в точке $a_{j}$, повьшая любое из нормирований в $a_{i}$. Так как монодромия диагональна, полученный базис снова будет либо левелевским, либо его перестановкой, и в нем $\Sigma_{1}=0, \Sigma_{2}=-1, \Sigma_{3}=1-$ опять уже рассмотренньй случай.

Докажем теперь реализуемость интересующей нас монодромии уравнением Римана с нужными суммами показателей. 
Пусть $\chi$ неприводима. Существует реализующая ее система такая, что $\Sigma_{1}=\Sigma_{2}=1$. Перейдя к уравнению путем повьшения нормирования в $a_{3}$, получим по следствию 1.2 искомое уравнение Римана. Если же $\chi$ приводима, то одна из $G_{i}$ должна быть диагонализуема и повышать нормирования надо именно в ней. Если диагональна $G_{3}$, то повторяется предыдущий случай; если же диагональна, например, $G_{2}$, поднимем $\varphi_{3}^{1}$, понижая одно из $\varphi_{2}^{j}$ (точнее, понизим $\varphi_{3}^{2}$, повысив любое из $\varphi_{2}^{j}$, после чего указанным в первой части леммы способом уменьшим на 2 сумму показателей в $a_{2}$ и повысим на 2 в $\left.a_{3}\right)$. Так как $G_{2}$ диагональна, то либо образ исходного левелевского в $a_{2}$ базиса, либо его перестановка будет левелевским базисом и, следовательно, можно повышать нормирование любого из столбцов. В любом случае по следствию 1.2 сумма показателей уравнения как в $a_{1}$, так и в $a_{2}$ будет равна единице. Таким образом, доказана следующая

Теорема 2.1. Всякое SL-представление, реализованное уравнением Римана, мохет быть реализовано и уравнением Римана-Штурма-Лиувилля.

Также можно отметить, что в важном для приложений частном случае - гипергеометрическом уравнении, условия на $S L$-монодромию имеют особенно простой вид. А именно, монодромия уравнения

$$
u^{\prime \prime}+\frac{\gamma-(1+\alpha+\beta) z}{z(z-1)} u^{\prime}-\frac{\alpha \beta}{z(z-1)} u=0
$$

лежит в $S L(2, \mathbb{C})$ тогда и только тогда, когда $\{\gamma, \alpha+\beta\} \in \mathbb{Z}$.

\section{3. $S L(2, \mathbb{Z})$-монодромия гипергеометрического уравнения}

Очевидно, всякая $S L(2, \mathbb{Z})$-монодромия, которая может быть реализована уравнением Римана и собственные значения которой хотя бы в двух матрицах единичны, может быть реализована гипергеометрическим уравнением. Встает вопрос, как по виду уравнения определить лежит ли его монодромия в $S L(2, \mathbb{Z})$. Ясно, что необходимым условием является принадлежность монодромии группе $S L(2, \mathbb{C})$. То есть в терминах коэффищиентов уравнения $\{\alpha+\beta, \gamma\} \in \mathbb{Z}$. Выясним будут ли эти условия достаточными. Во-первых, очевидно, что для приводимых представлений этих условий достаточно. Действительно, всякая приводимая монодромия гипергеометрического уравнения приводится к виду

$$
G_{0}=\left(\begin{array}{ll}
1 & c \\
0 & 1
\end{array}\right), \quad G_{1}=\left(\begin{array}{ll}
1 & 0 \\
0 & 1
\end{array}\right), \quad G_{\infty}=G_{1}^{-1} G_{0}^{-1} .
$$

Следовательно, после сопряжения на $S_{0}=\operatorname{diag}\left(c^{-1}, 1\right)$ получаем

$$
G_{0}=\left(\begin{array}{ll}
1 & 1 \\
0 & 1
\end{array}\right), \quad G_{1}=\left(\begin{array}{ll}
1 & 0 \\
0 & 1
\end{array}\right), \quad G_{\infty}=G_{1}^{-1} G_{0}^{-1}
$$

В случае неприводимого представления требуется более тщательное рассмотрение. Известно, что всякое неприводимое представление монодромии уравнения Римана $\chi$ в некотором базисе имеет вид

$$
G_{0}=\left(\begin{array}{ll}
1 & 0 \\
d & 1
\end{array}\right), \quad G_{1}=\left(\begin{array}{ll}
1 & c \\
0 & 1
\end{array}\right), \quad G_{\infty}=G_{1}^{-1} G_{0}^{-1}, \quad c, d \in \mathbb{C} .
$$


Сопрягая на матрицу $S_{1}=\operatorname{diag}(d, 1)$, получаем

$$
G_{0}=\left(\begin{array}{ll}
1 & 0 \\
1 & 1
\end{array}\right), \quad G_{1}=\left(\begin{array}{cc}
1 & c d \\
0 & 1
\end{array}\right), \quad c d \in \mathbb{C}
$$

Теперь сопряжение на произвольную $S_{2} \in S L(2, \mathbb{C})$ дает

$$
G_{0}=\left(\begin{array}{cc}
1+\mu \tau & -\mu^{2} \\
\tau^{2} & 1-\mu \tau
\end{array}\right), \quad G_{1}=\left(\begin{array}{cc}
1-\lambda \nu b & \lambda^{2} b \\
-\nu^{2} b & 1+\lambda \nu b
\end{array}\right), \quad b \in \mathbb{C},
$$

где

$$
S_{2}=\left(\begin{array}{cc}
\lambda & \mu \\
\nu & \tau
\end{array}\right)
$$

Таким образом, критерием возможности приведения к $S L(2, \mathbb{Z})$ является существование набора $\{\lambda, \mu, \nu, \tau\}$, удовлетворяющего

$$
\left\{\begin{array}{l}
\mu^{2}, \tau^{2}, \quad \mu \tau \in \mathbb{Z}, \\
\lambda^{2} b, \nu^{2} b, \quad \lambda \nu b \in \mathbb{Z}, \\
\lambda \tau-\mu \nu=1
\end{array}\right.
$$

Очевидно, если он существует, то

$$
\lambda^{2} \tau^{2} b, \mu^{2} \nu^{2} b, \lambda \mu \nu \tau b \in \mathbb{Z} \Longrightarrow\left(\lambda^{2} \tau^{2}+\mu^{2} \nu^{2}-2 \lambda \mu \nu \tau\right) b=(\lambda \tau-\mu \nu)^{2} b=b \in \mathbb{Z} .
$$

Заметим, что $b=\operatorname{tr} G_{\infty}-2=e^{2 \pi i \alpha}+e^{2 \pi i \beta}-2$. Таким образом, получаем

УТВЕРЖДЕНИЕ 3.1. Монодромия гипергеометрического уравнения лежит $в$ $S L(2, \mathbb{Z})$, если и только если выполняется $\left\{\gamma, \alpha+\beta, e^{2 \pi i \alpha}+e^{2 \pi i \beta}\right\} \in \mathbb{Z}$.

Полученные условия можно рассмотреть более подробно. Имеем $e^{2 \pi i \alpha}+e^{2 \pi i \beta}=e^{2 \pi i \alpha}\left(1+e^{2 \pi i(\alpha+\beta-2 \alpha)}\right)=e^{2 \pi i \alpha}+e^{-2 \pi i \alpha}=e^{2 \pi i \beta}+e^{-2 \pi i \beta}=k, \quad k \in \mathbb{Z}$. Таким образом, для $x=e^{2 \pi i \alpha}$ получаем $x+x^{-1}=k$. Вместе с условием $(\alpha+\beta) \in \mathbb{Z}$ это влечет $e^{2 \pi i \alpha}=\left(k+\sqrt{k^{2}-4}\right) / 2, e^{2 \pi i \beta}=\left(k-\sqrt{k^{2}-4}\right) / 2$.

Окончательно, монодромия гипергеометрического уравнения лежит в $S L(2, \mathbb{Z})$ при следующих значениях показателей:

$$
\alpha=\frac{1}{2 \pi i} \ln \frac{k \pm \sqrt{k^{2}-4}}{2}, \quad \beta=-\alpha=\frac{1}{2 \pi i} \ln \frac{k \mp \sqrt{k^{2}-4}}{2}, \quad \gamma=l, \quad k, l \in \mathbb{Z} .
$$

Уравнение, таким образом, имеет вид

$$
u^{\prime \prime}+\frac{l-z}{z(z-1)} u^{\prime}-\frac{\frac{1}{(2 \pi)^{2}}\left(\ln \frac{k \pm \sqrt{k^{2}-4}}{2}\right)^{2}}{z(z-1)} u=0
$$

где $k, l$ целочисленны. 


\section{СПИСОК ЦИТИРОВАННОЙ ЛИТЕРАТУРЫ}

[1] Iwasaki K., Kimura H., Shimomura Sh., Yoshida M. From Gauss to Painlevé. A Modern Theory of Special Functions. Aspects of Math. V. E16. Braunschweig: Friedr. Vieweg \& Sohn, 1991.

[2] Kimura T., Shima K. A note on the monodromy of the hypergeometric differential equation // Japan J. Math. 1991. V. 17. P. 137-163.

[3] Болибрух А. А. О достаточных условиях положительнй разрешимости проблемы Римана-Гильберта // Матем. заметки. 1992. Т. 51. № 2. С. 9-19.

[4] Зограф П.Г., Тахтаджян Л.А. Об уравнении Лиувилля, акцессорных параметрах и геометрии пространства Тейхмюллера для римановых поверхностей рода 0 // Матем. сб. 1987. T. 132 (174). № 2. C. 147-166.

[5] Форстер О. Римановы поверхности. М.: Мир, 1980.

[6] Болибрух А. А. 21-я проблема Гильберта для линейных фуксовых систем. Тр. МИАН. T. 206. М.: Наука, 1994.

[7] Levelt A. H. M. Hypergeometric functions // Indag. Math. 1961. V. 23. P. 361-403.

[8] Голубев В.В.Лекции по аналитической теории дифференциальных уравнений. М.: Гостехтеоретиздат, 1950.

[9] Крылов Б. Л. Решение в конечном виде проблемы Римана для системы Гаусса // Тр. Казан. авиац. ин-та. 1956. Т. 31.

[10] Dekkers W. The matrix of a connection having regular singularities on a vector bundle of rank 2 on $P^{1}(C) / /$ Lecture Notes in Math. V. 712. Berlin: Springer, 1979. P. 33-43.

Математический институт им. В. А. Стеклова РАН

Поступило

E-mail: poberezh@itep.ru 